were evaluated and compared with the theoretical energy requirements.

Results Mean caloric intake on the first day was 59\% (SD \pm 46.4 ) and on the second day was $64.3 \%(\mathrm{SD} \pm 47.4)$ of daily requirements. $72(57.1 \%)$ patients received $\geq 80 \%$ of required daily calories, without a significant difference with patients who received $<80 \%$ of daily calories, $(\mathrm{p}=0.3)$.

The overall mortality rate was $28.5 \%$. Patients who received $<80 \%$ of daily caloric needs were 4 times more likely to have a fatal outcome, compared to those who received $\geq 80 \%$ of daily caloric needs [OR=4.0 95\% CI (1.2-12.7) $\mathrm{p}=0.01]$.

Daily caloric intake of $\geq 80 \%$ resulted a protective factor against death in the Cox proportional-hazard regression model $(b=-1.1$, $\mathrm{p}=0.02$ ).

Conclusions We have to increase the number of patients who receive $\geq 80 \%$ of daily caloric requirements and provide appropriate nutritional support during the first days of admission. Mortality rate remains high, due to the large number of patients receiving $<80 \%$ of needed calories.

\section{MEDICAL RADIATION EXPOSURE IN CHILDREN DIAGNOSED WITH ACUTE LYMPHOBLASTIC LEUKEMIA FROM 1995- 2010: A SINGLE INSTITUTION STUDY}

doi:10.1136/archdischild-2012-302724.1464

R Robin Rohrer. Epidemiology, University of Pittsburgh, Pittsburgh, PA, USA

Objective This retrospective study examines a cohort of children diagnosed with acute lymphoblastic leukemia, examining exposure to medical radiation pre-conception, pre-natal or in early childhood. Exposure is documented through family interview. The study encompasses children diagnosed with A.L.L. and treated at the Children's Hospital of Pittsburgh over a fifteen year period.

Background Early exposure to medical radiation is one of the identified risks for childhood leukemias but documentation is difficult and mostly lacking in the United States experience. The author of this study developed a questionnaire that examines radiation exposures in either parent of to the child later diagnosed.

Methods Each family who was consented to be interviewed completed a five page questionnaire at clinic visit, through phone or mail. Whenever possible both parents were interviewed.

Results To date the author has been able to interview about $70 \%$ of children diagnosed from 2005-2010 however the interview rate for the period 1990-2005 is approximately at 5\%. Among the families interviewed at least one exposure was commonly documented.

Conclusions Exposure to medical radiation for a child later diagnosed with A.L.L. may at occur at several critical junctures. Chest or sinus x-rays or CT of a parent pre-conception, particularly repeated scans have the possibility of DNA damage. Early childhood exposure through the diagnostic process (ruling out infection or trauma) may well contribute to this "perfect storm" in the still elusive causes of childhood A.L.L.

\section{CLINICAL CHARACTERISTICS AND TREATMENT RESULTS OF NEUROBLASTOMA PATIENTS}

doi:10.1136/archdischild-2012-302724.1465

G Tokuç. Marmara University, Istanbul, Turkey

Objective and method: The survival of the patients with neuroblastoma has improved in last few decades. But still it depends on various clinical and biological factors. To assess the clinical features and trends in survival, the data for 56 newly diagnosed patients between September 1996 and August 2011 from a single center were retrospectively analyzed.
Results Histopathologic subtypes were neuroblastoma (NBL) in 52 patients and ganglioneuroblastoma in 4 patients. The median age was 2.5 years and Male/Female ratio was $1.2 / 1$. Primary tumor sites were abdomen, thorax, and neck with the frequency of $77.4 \%$, $19 \%$ and $3.6 \%$ respectively. There were $21,22,9,3.1$ patients with stage $1,2,3,4,4 \mathrm{~S}$ disease and their 5 -year survival rates were $100 \%$, $74 \%, 33 \%, 6.9 \%$, and $59 \%$, respectively In multivariate analysis, stage 4 disease $(P<0.001)$, abdominal primary tumor site $(P<0.001)$, NBL subtype in histopathology $(P=0.001)$, and responsiveness to chemotherapy $(P<0.001)$ were the determinants of poor prognosis. Conclusions The survival rates in children with local disease are comparable with the results of developed countries; however, the results in children with advanced disease are still not satisfactory. To improve the outcome, especially in children with advanced disease, more effective chemotherapy regimens and molecular therapies should be investigated. Sharing the knowledge and capacity building to improve the treatment results in NBL are also critical for developing countries.

\section{THE ETIOLOGY OF PERIPHERAL LYMPHADENOPATHY IN CHILDREN \\ doi:10.1136/archdischild-2012-302724.1466}

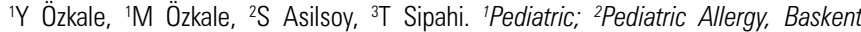
University, Adana; ${ }^{3}$ Pediatric Nephrology, Ufuk University, Ankara, Turkey

Aim To determine the clinical and laboratory findings of peripheral lymphadenopathies.

Methods We evaluated 224 children who had been admitted to our pediatricclinic becauseofenlarged peripherallymphadenopathy(LAP). The patients age, sex, localization, duration, size, mobility, extansion of lymph nodes, systemic symptoms, diagnostic laboratory and radiologic investigations and final diagnosis were determined. Features of lympadenopathy were compared between patients with malignant and benign diagnosis.

Results The ages of the patients ranged between 2 months and 16 years (median 7 years); 56\% of the patients were male, Of the 186 (83\%) patient had benign and $38(17 \%)$ had malignant disorder. A total of $164(73 \%)$ had localized, 60 (27\%) had generalized LAP. The most frequent cause in the benign group was acute lymphadenitis where as hodgkin lymphoma was most frequent in the malignant group. Cervical region was the most common localization for benign or malign disorder. The mean age was higher in the malignant group. We determined acute LAP in 164 (73\%) and choronic LAP in $60(27 \%)$ patient. We didn't find differences between the benign and malignant groups according to size and mobility of nodes. Anemia, leukopenia trombocytopenia and organomegaly were significantly common in malignant group. Weight loss, night sweet and fatigue were more frequently associated symptoms in the malignant group. Excisional biopsies were performed to 50 (22.3\%) patient.

Conclusion The following findings were important to alert the physician about the probability of a malign disorder: older age, supraclavicular lymphadenopathy, abdominal LAP, abnormal complete blood count and organomegaly.

\section{PEDIATRIC ONCOLOGY PATIENTS PRESENTING WITH SPINAL CORD COMPRESSION}

doi:10.1136/archdischild-2012-302724.1467

G Tokuç. Marmara University, Istanbul, Turkey

Spinal cord disease in children with known or suspected malignancy is an oncological emergency because it commonly implies malignant spinal cord compression. The records of 17 children with cancer presenting with spinal cord compression, encountered over 